\title{
CORRECTION
}

Praneeth Nampally • J. N. Reddy 10

\section{Correction to: Geometrically nonlinear Euler-Bernoulli and Timoshenko micropolar beam theories}

Published online: 10 October 2020

(C) Springer-Verlag GmbH Austria, part of Springer Nature 2020

Correction to: Acta Mech 231, 4217-4242 (2020)

https://doi.org/10.1007/s00707-020-02764-x

In the version of the article originally published, Eq. (5), Eq. (54), the last equation of equation set (66), and the last equation of equation set (67) were shown incorrectly.

Equation (5):

$$
\psi=\psi_{1} \hat{\mathbf{e}}_{1}+\psi_{2} \hat{\mathbf{e}}_{2}+\psi_{3} \hat{\mathbf{e}}_{3}
$$

Equation (54):

$$
-\frac{\mathrm{d} \mathfrak{M}_{x x}^{E(s)}}{\mathrm{d} x}+\mathfrak{N}_{x x}^{E(s)} \psi_{y}^{E}+2 \mathfrak{D}_{x}^{E(a)}=0
$$

Last equation of equation set (66):

$$
0=\int_{x_{a}}^{x_{b}}\left\{E_{44} \frac{\mathrm{d} w_{4}}{\mathrm{~d} x} \frac{\mathrm{d} \psi_{y}^{\mathrm{T}}}{\mathrm{d} x}-A_{77} w_{4}\left(\phi_{x}^{\mathrm{T}}-\frac{\mathrm{d} w_{0}^{\mathrm{T}}}{\mathrm{d} x}-2 \psi_{y}^{\mathrm{T}}\right)\right\} \mathrm{d} x-Q_{7} w_{4}\left(x_{a}\right)-Q_{8} w_{4}\left(x_{b}\right)
$$

Last equation of equation set (67):

$$
\begin{aligned}
0= & \int_{x_{a}}^{x_{b}}\left\{E_{44} \frac{\mathrm{d} w_{4}}{\mathrm{~d} x} \frac{\mathrm{d} \psi_{y}^{\mathrm{T}}}{\mathrm{d} x}-A_{11} w_{4} \frac{\mathrm{d} w_{0}^{\mathrm{T}}}{\mathrm{d} x}\left(\frac{\mathrm{d} u_{0}^{\mathrm{T}}}{\mathrm{d} x}-\psi_{y}^{\mathrm{T}} \frac{\mathrm{d} w_{0}^{\mathrm{T}}}{\mathrm{d} x}\right)\right. \\
& \left.-A_{77} w_{4}\left(\phi_{0}^{\mathrm{T}}-\frac{\mathrm{d} w_{0}^{\mathrm{T}}}{\mathrm{d} x}-2 \psi_{y}^{\mathrm{T}}\right)\right\} \mathrm{d} x-Q_{7} w_{4}\left(x_{a}\right)-Q_{8} w_{4}\left(x_{b}\right)
\end{aligned}
$$

These corrections do not affect any of the results or other expressions reported in the paper.

Publisher's Note Springer Nature remains neutral with regard to jurisdictional claims in published maps and institutional affiliations.

The original article can be found online at https://doi.org/10.1007/s00707-020-02764-x.

P. Nampally · J. N. Reddy ( $\square)$

Department of Mechanical Engineering, Texas A\&M University, College Station, TX 77843-3123, USA

E-mail: jnreddy@tamu.edu 